\title{
Farinha de resíduos de feijoa (Acca sellowiana): caracterização química, aceitação sensorial e intenção de compra de biscoitos tipo cookies
}

\author{
Feijoa (Acca sellowiana) wastes flour: chemical characterization, sensory acceptance and purchase \\ intention of cookies
}

Harina de residuos de fijoa (Acca sellowiana): caracterización química, aceptación sensorial y intención de compra de cookies

Recebido: 07/09/2021 | Revisado: 15/09/2021 | Aceito: 18/09/2021 | Publicado: 19/09/2021

Patricia Muniz de Oliveira
ORCID: https://orcid.org/0000-0002-5868-2011
Instituto Federal de Educação, Ciência e Tecnologia de Santa Catarina, Brasil
E-mail: patriciaurupema@ gmail.com
Ana Carolina Moura de Sena Aquino
ORCID: https://orcid.org/0000-0002-0832-5198
Instituto Federal de Educação, Ciência e Tecnologia de Santa Catarina, Brasil
E-mail: ana.carolina@ifsc.edu.br

\begin{abstract}
Resumo
O objetivo deste estudo foi realizar a caracterização físico-química da farinha de resíduos do despolpamento da feijoa (FRF) e verificar a potencialidade da sua utilização em biscoitos tipo cookies, por meio de propriedades tecnológicas e aceitação sensorial. A FRF foi obtida a partir da secagem a $55{ }^{\circ} \mathrm{C}$ e da moagem dos resíduos (endocarpo e mesocarpo) da feijoa, sendo caracterizada físico-quimicamente (composição centesimal, pH, ATT, carotenoides totais, ácido ascórbico e composição mineral) e utilizada na elaboração de cookies com substituição parcial de farinha de trigo (F1-10\%FRF e F2-20\%FRF), com posterior análise de características tecnológicas e teste de aceitação sensorial. A FRF se destacou em relação ao seu teor de fibra alimentar total $(40,50 \mathrm{~g} / 100 \mathrm{~g})$ e se mostrou uma importante e potencial fonte de elementos essenciais. Os cookies com FRF tiveram maiores médias de aceitação global, sendo que a amostra F2 apresentou o maior índice de aceitabilidade $(88,17 \%)$. Todas as médias das notas atribuídas pelos provadores no teste de aceitabilidade, para as amostras F1 e F2, foram próximas de 8 na escala hedônica, demonstrando que os provadores "gostaram muito". O teste de intenção de compra reproduziu a aceitabilidade dos provadores, sendo que as médias das notas para as amostras corresponderam a "certamente compraria" e "provavelmente compraria" na escala hedônica de intenção de compra. Os resultados obtidos neste estudo evidenciam a potencialidade da utilização da FRF, podendo contribuir para a diversificação da oferta de produtos, além de agregar valor ao resíduo do processamento de uma fruta nativa com potenciais características nutricionais.
\end{abstract}

Palavras-chave: Goiaba serrana; Resíduo de fruta; Análise físico-química; Análise sensorial; Biscoito.

\begin{abstract}
The aim of this study was to carry out the physical-chemical characterization of the feijoa residue flour (FRF) and to verify the potential of its use in cookies, through its technological properties and sensory acceptance. The FRF was obtained from drying at $55{ }^{\circ} \mathrm{C}$ and grinding the residues (endocarp and mesocarp) of the feijoa, being physicallychemically characterized (centesimal composition, pH, ATT, total carotenoids, ascorbic acid and mineral composition) and in the preparation cookies with partial replacement of wheat flour (F1-10\% FRF and F2-20\% FRF), with subsequent analysis of technological characteristics and sensory acceptance test. FRF stood out in relation to its total dietary fiber content $(40.50 \mathrm{~g} / 100 \mathrm{~g})$ and proved to be an important and potential source of essential elements. Cookies with FRF had the highest averages of global acceptance, and the F2 sample had the highest acceptability rate $(88.17 \%)$. All the averages of the scores assigned by the tasters in the acceptability test, for samples F1 and F2, were close to 8 on the hedonic scale, demonstrating that the tasters "liked it a lot". The purchase intention test reproduced the acceptability of the tasters, with the mean scores for the samples corresponding to "certainly buy" and "probably buy" on the hedonic scale of purchase intention. The results obtained in this study show the potential of using FRF, which can contribute to the diversification of the offer of products, in addition to adding value to the residue from the processing of a native fruit with potential nutritional characteristics.
\end{abstract}

Keywords: Feijoa; Fruit by-product; Chemical physical analysis; Sensory analysis; Cookie. 


\section{Resumen}

El objetivo de este estudio fue realizar la caracterización fisicoquímica del residuo de fijoa (FRF) y verificar el potencial de su uso en cookies, a través de sus propiedades tecnológicas y aceptación sensorial. El FRF se obtuvo del secado a $55^{\circ} \mathrm{C}$ y moliendo los residuos (endocarpio y mesocarpio) del fijoa, siendo caracterizado fisicoquímicamente (composición centesimal, pH, ATT, carotenoides totales, ácido ascórbico y composición mineral) y utilizado en la preparación de cookies con sustitución parcial de harina de trigo (F1-10\% FRF y F2-20\% FRF), con posterior análisis de características tecnológicas y prueba de aceptación sensorial. FRF se destacó en relación con su contenido total de fibra dietética (40,50 g / $100 \mathrm{~g})$ y demostró ser una fuente importante y potencial de elementos esenciales. Las cookies con FRF tuvieron los promedios más altos de aceptación global y la muestra F2 tuvo la tasa de aceptabilidad más alta $(88,17 \%)$. Todas las medias de las puntuaciones asignadas por los catadores en la prueba de aceptabilidad, para las muestras F1 y F2, se acercaron a 8 en la escala hedónica, demostrando que a los catadores "les gustó mucho". La prueba de intención de compra reprodujo la aceptabilidad de los catadores, con las puntuaciones medias de las muestras correspondientes a "comprar ciertamente" y "comprar probablemente" en la escala hedónica de intención de compra. Los resultados obtenidos en este estudio muestran el potencial del uso de FRF, que puede contribuir a la diversificación de la oferta de productos, además de agregar valor al residuo de procesamiento de una fruta nativa con potenciales características nutricionales.

Palabras clave: Fijoa; Residuos de frutas; Análisis físico químico; Análisis sensorial; Galleta.

\section{Introdução}

A feijoa (Acca sellowiana), também conhecida como goiaba-serrana, é uma fruta da família Myrtaceae e nativa da América do Sul (sul do Brasil, norte da Argentina e oeste do Paraguai e Uruguai) (Arioli et al., 2018). Apesar de ser pouco conhecida no Brasil, é uma fruta cultivada em várias regiões do mundo e apreciada pelos seus sabor e aroma únicos (Amarante et al., 2019). Na Nova Zelândia e na Austrália seu cultivo já é popular, sendo exportada como fruta fresca ou produtos industrializados como sucos, bebidas alcoólicas, geleias, compotas, doces e outros (González-García et al., 2017).

Benefícios à saúde vem sendo associados a presença de compostos bioativos na feijoa, como concentrações significativas de polifenois, carotenoides e vitaminas, além do seu destaque pela ação antimicrobiana, antitumoral, antinflamatória, antioxidante, gastroprotetora e hepatoprotetora (Weston, 2010; Monforte et al., 2014; Amarante et al., 2017b).

Dentre os diversos subprodutos, o aproveitamento de resíduos de frutas pode resultar em benefícios econômicos e impactar positivamente no meio ambiente (Ayala-Zavala et al., 2011). Devido ao seu baixo rendimento em polpa (endocarpo), há uma necessidade de alternativas para aproveitamento do mesocarpo e epicarpo (casca) da feijoa, que geralmente são descartados no seu processamento. A polpa da feijoa se caracteriza por ser suculenta e doce, enquanto sua casca, que representa $50 \%$ do peso do fruto, tem uma característica ácida e amarga (Santos et al., 2019).

Alguns estudos reportam a potencialidade do aproveitamento da casca de feijoa pelos seus expressivos teores de fibras, vitaminas, compostos fenólicos e suas atividades antioxidante e antibacteriana, incluindo pesquisa com a incorporação desse resíduo em embalagens biodegradáveis (Sun-Waterhouse et al., 2013; Santos et al., 2019; Almeida et al., 2020; Sganzerla et al., 2020).

O processamento industrial da feijoa e o seu consumo in natura resultam em quantidades consideráveis de resíduos, os quais apresentam relevantes concentrações de compostos bioativos, os quais podem ser benéficos para a saúde humana (Sun-Waterhouse et al., 2013). O uso desses resíduos representa uma oportunidade aproveitamento integral da matéria-prima, além de produzir ingredientes com valor agregado para aplicação em produtos alimentícios e permitir a oferta de produtos com feijoa fora da estação, já que a mesma é uma cultura sazonal com curta temporada de colheita.

Uma das alternativas viáveis para a utilização dos resíduos de frutas é através de sua transformação em farinha, reduzindo a atividade de água, as reações químicas e microbiológicas e aumentando a sua vida útil, tornando uma opção viável para aplicações tecnológicas, inclusive em produtos de panificação (Lima et al., 2015; Soquetta et al., 2016).

Diante disso, o objetivo deste estudo foi realizar a caracterização físico-química da farinha de resíduos do despolpamento da feijoa e verificar a potencialidade da sua utilização em biscoitos tipo cookies, por meio de propriedades tecnológicas, aceitação sensorial e intenção de compra. 


\section{Metodologia}

\subsection{Obtenção da farinha de resíduos de feijoa (FRF)}

As amostras de feijoa foram provenientes da Estação Experimental da Empresa de Pesquisa Agropecuária e Extensão Rural de Santa Catarina (EPAGRI) da cidade de São Joaquim-SC (28¹6’40.02” S, 4956’09.10” W e altitude de 1400 m), na safra de 2019. Após a colheita, as frutas foram transportadas em caixa de polietileno até o Laboratório de Processamento de Frutas e Hortaliças do Instituto Federal de Santa Catarina, Câmpus Urupema, onde foram processadas. Primeiramente, as amostras foram higienizadas com água corrente e sanitizadas com hipoclorito de sódio a 200 ppm por 15 minutos. Em seguida, as frutas foram cortadas ao meio com facas de aço inox e a polpa foi manualmente separada da casca (endocarpo) e do mesocarpo, considerados como resíduos para fins deste estudo.

Para obtenção da farinha, o resíduo do processamento da feijoa foi cortado em pequenas fatias $(0,5 \mathrm{~cm}$ de espessura), as quais foram dispostas em bandeja para posterior secagem. A secagem foi realizada em estufa com circulação forçada de ar (Adamo®, modelo 310/81PID) a $55^{\circ} \mathrm{C}$, por aproximadamente 24 horas. Após a secagem, o resíduo foi moído em moinho de facas tipo willey (Fortinox®, modelo STAR FT 50, Piracicaba, Brasil), com tamanho de partícula de 400 mesh e armazenado em recipiente de vidro, hermeticamente fechado, até o momento das análises e da sua utilização.

\subsection{Caracterização físico-química da farinha de resíduos de feijoa}

A farinha de resíduos de feijoa foi caracterizada quanto aos teores de: umidade $(\mathrm{g} / 100 \mathrm{~g})$ pelo método gravimétrico de volatilização por secagem direta em estufa a $105{ }^{\circ} \mathrm{C}$, de proteínas (g/100g) por meio da técnica de Kjeldahl a partir da determinação de nitrogênio total, de lipídeos totais $(\mathrm{g} / 100 \mathrm{~g})$ através da extração direta em Soxhlet e de cinzas (g/100g) por incineração em mufla a $550{ }^{\circ} \mathrm{C}$ (IAL, 2008). O teor de fibra alimentar total (g/100g) foi determinado pelo método enzimáticogravimétrico AOAC 991.43 (AOAC, 2005) e o teor de carboidratos totais foi calculado por diferença [100 - (umidade + cinzas + proteínas + lipídeos)].

A determinação do $\mathrm{pH}$ se deu através de potenciômetro, enquanto a acidez titulável em ácido orgânico (g de ácido cítrico/100g) foi determinada segundo metodologia do IAL (2008).

O teor de ácido ascórbico foi determinado pelo método padrão, n. 43.065, da AOAC (1984) modificado por Benassi e Antunes (1988), no qual se substitui o solvente extrator ácido metafosfórico por ácido oxálico, que se baseia na redução de 2,6diclorofenolindofenol-sódico (DCFI) pelo ácido ascórbico.

Os carotenoides totais foram determinados conforme metodologia de Rodriguez-Amaya (1999), determinando a absorbância em $450 \mathrm{~nm}$ do extrato obtido, utilizando um espectrofotômetro (U-1800, Hitachi, Tóquio, Japão), sendo a concentração $(\mu \mathrm{g} / 100 \mathrm{~g})$ calculada se empregando o coeficiente de absorção $\left(\mathrm{A}^{1 \%}{ }_{1 \mathrm{~cm}}\right)$ do $\beta$-caroteno em éter de petróleo.

A FRF foi avaliada quanto aos teores totais $(\mathrm{mg} / \mathrm{kg})$ dos macronutrientes cálcio $(\mathrm{Ca})$, magnésio $(\mathrm{Mg})$, potássio $(\mathrm{K})$, nitrogênio $(\mathrm{N})$, fósforo $(\mathrm{P})$ e enxofre $(\mathrm{S})$, e dos micronutrientes ferro $(\mathrm{Fe})$, cobre $(\mathrm{Cu})$, zinco $(\mathrm{Zn})$ e manganês $(\mathrm{Mn})$. O teor de $\mathrm{N}$ foi avaliado pelo método Kjeldahl, o teor de $\mathrm{P}$ por espectrofotometria, o teor de $\mathrm{S}$ por turbidimetria, o teor de $\mathrm{K}$ por fotometria de chama e os teores de $\mathrm{Ca}, \mathrm{Mg}, \mathrm{Fe}, \mathrm{Cu}, \mathrm{Zn}$ e $\mathrm{Mn}$ por espectrofotometria de absorção atômica (Silva, 2009).

Todas as análises foram realizadas em triplicata $(n=3)$, sendo os resultados expressos como média e desvio padrão.

\subsection{Elaboração dos cookies}

A Tabela 1 apresenta as formulações dos biscoitos tipo cookies elaborados com substituição parcial da farinha de trigo pela farinha de resíduos de feijoa (FRF) nas concentrações de $10 \%$ e $20 \%$.

Para produção dos cookies, primeiramente misturou-se os ingredientes secos (açúcar refinado, açúcar demerara, sal e fermento químico). Separadamente foram misturados os ovos, a manteiga e a essência de baunilha até obtenção de um creme 
homogêneo e, aos poucos, foi adicionado o mix de farinhas e a mistura de ingredientes secos ao creme. A massa foi homogeneizada em uma batedeira planetária (KitchenAid, Artisan) e os biscoitos foram moldados (aro de $5 \mathrm{~cm}$ ) e colocados para assar em forno elétrico (Wictory, WC-03N) a $160{ }^{\circ} \mathrm{C}$ por 17 minutos. Em seguida, os cookies foram resfriados a temperatura ambiente e armazenados em potes de vidro com tampa hermética.

Tabela 1. Formulações dos cookies com substituição parcial da farinha de trigo por farinha de resíduos do despolpamento de feijoa.

\begin{tabular}{lccc}
\hline $\begin{array}{l}\text { Ingredientes } \\
\text { (g/100g de farinha de trigo) }\end{array}$ & $\begin{array}{c}\text { Padrão } \\
(0 \% \text { FRF })\end{array}$ & $\begin{array}{c}\text { F1 } \\
(10 \% \text { FRF })\end{array}$ & $\begin{array}{c}\text { F2 } \\
(20 \% \text { FRF })\end{array}$ \\
\hline Farinha de trigo refinada & 125,0 & 112,5 & 100,0 \\
Farinha de trigo integral & 125,0 & 112,5 & 100,0 \\
Farinha de resíduos de feijoa (FRF) & 0,0 & 25,0 & 50,0 \\
Açúcar refinado & 47,5 & 47,5 & 47,5 \\
Açúcar demerara & 67,5 & 67,5 & 67,5 \\
Sal & 1,0 & 1,0 & 1,0 \\
Ovo & 47,5 & 47,5 & 47,5 \\
Manteiga sem sal & 75,0 & 75,0 & 75,0 \\
Fermento químico & 5,0 & 5,0 & 5,0 \\
Essência de baunilha & 3,5 & 3,5 & 3,5 \\
\hline
\end{tabular}

Fonte: Adaptado de Diettrich, Bauer, Oliveira (2016) pelos autores (2021).

\subsection{Características tecnológicas dos cookies}

Os cookies foram pesados antes e após o forneamento para determinação da perda de massa (\%), a espessura e o diâmetro após o forneamento (uma hora após esse processo) foram medidos com um paquímetro digital e o fator de expansão foi calculado pela razão entre o diâmetro e a espessura dos cookies após o forneamento (AACC, 2000).

\subsection{Determinação da cor dos cookies}

Para a avaliação da cor instrumental dos cookies utilizou-se colorímetro calibrado Delta Color, modelo Delta Vista 450G, sendo obtidos os parâmetros $\mathrm{L}^{*}$ que indica a luminosidade (claro/escuro); a* para a cromaticidade no eixo da cor verde (-) para vermelha (+); e b* que indica a cromaticidade no eixo da cor azul (-) para amarela (+) do sistema CIELAB. A diferença de cor $\left(\Delta \mathrm{E}^{*}\right)$ dos cookies foi calculada pela equação $\Delta \mathrm{E}^{*}=\left(\left(\Delta \mathrm{L}^{* 2}\right)+\left(\Delta \mathrm{a}^{* 2}\right)+\left(\Delta \mathrm{b}^{* 2}\right)\right)^{1 / 2}$ em relação à formulação padrão.

\subsection{Análise sensorial}

Para a análise sensorial, o estudo foi previamente submetido e aprovado pelo Comitê de Ética em Pesquisa com Seres Humanos da Universidade do Planalto Catarinense, mediante parecer $n^{\circ}$ 3.637.408. Foram realizados testes de aceitação e de intenção de compra com 55 provadores de ambos os sexos (65,5\% feminino e 34,5\% masculino), com idade entre 18 e 56 anos, não treinados, pertencentes à comunidade acadêmica do IFSC Campus Urupema, que aderiram livremente à pesquisa, mediante assinatura do Termo de Consentimento Livre e Esclarecido (TCLE).

Para o teste de aceitabilidade, os cookies foram oferecidos em ordem aleatória e de forma monádica, para que avaliassem os atributos de aparência, aroma, sabor, textura e aceitação global, a partir de escala hedônica estruturada de nove pontos, sendo 1 para "desgostei muitíssimo" e 9 para "gostei muitíssimo". 
A partir das médias do teste de aceitabilidade, calculou-se o índice de aceitabilidade (IA) através da seguinte fórmula: $\mathrm{IA}=(\mathrm{A} \times 100) / \mathrm{B}$, em que $\mathrm{A}=$ média das notas atribuídas pelos provadores e $\mathrm{B}=$ nota máxima atribuída ao produto.

$\mathrm{O}$ teste de intenção de compra foi realizado a partir de escala hedônica estruturada de cinco pontos, sendo 1 para "certamente compraria" e 5 para "certamente não compraria".

\subsection{Análise estatística}

Os resultados de todas as análises foram expressos como média e desvio padrão. Os dados foram submetidos à análise estatística de ANOVA, teste de comparação de médias de Tukey ao nível 5\% de probabilidade pelo software Statistica 10.

\section{Resultados e Discussão}

Na Tabela 2 estão descritos os resultados da caracterização da farinha de resíduos de feijoa (Figura 1). Observa-se que o teor de umidade atende ao previsto na legislação brasileira para farinhas (máximo de 15\%) (Brasil, 2005). Amarante et al. (2019), caracterizando a casca de diferentes genótipos de feijoa in natura, encontraram valores de cinzas variando de 0,30 a $1,49 \mathrm{~g} / 100 \mathrm{~g}$, sendo que a farinha em estudo apresentou $2,31 \mathrm{~g} / 100 \mathrm{~g}$.

Figura 1. Resíduos (epicarpo e mesocarpo) da feijoa: (A) após secagem a $55^{\circ} \mathrm{C} / 24$ horas e (B) após secagem a $55^{\circ} \mathrm{C} / 24$ horas e moagem (400 mesh) - FRF.
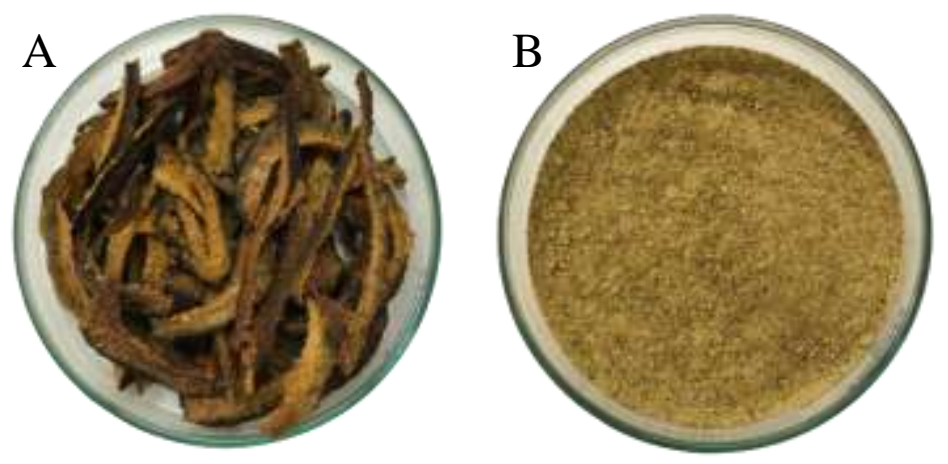

Fonte: Autores (2021).

Os teores de lipídeos totais e de proteína bruta da FRF foram, respectivamente, 1,27 e 3,70 g/100g. Esses valores são superiores aos encontrados por Almeida et al. (2020) para a farinha obtida apenas do mesocarpo da feijoa, o que sugere que o epicarpo contribui para o incremento de proteínas e de lipídeos na farinha. Neste último, justificado pelos óleos essenciais presentes no epicarpo, conforme relatado por Zhu (2018), que também afirma que a fruta in natura é caracterizada por possuir teores relativamente baixos de lipídeos e proteínas.

Em relação ao teor de fibra alimentar total, observa-se a representatividade $(40,50 \mathrm{~g} / 100 \mathrm{~g})$ desse componente na farinha em estudo, reforçando a sua potencialidade como um ingrediente para o desenvolvimento de produtos com alegação de propriedades funcionais possibilitando assim benefícios à saúde. Segundo a RDC n 54 de 12 de novembro de 2012 da ANVISA (Brasil, 2012), que dispõe sobre o Regulamento Técnico sobre Informação Nutricional Complementar, para um produto possuir alegação de alto teor ou rico em fibras deve conter no mínimo $5 \mathrm{~g}$ de fibra por porção. A RDC n ${ }^{\circ} 359$ de 23 de dezembro de 2003 da ANVISA (Brasil, 2003), não estabelece porção para farinhas de frutas, mas considerando a porção definida para frutas desidratadas ( $50 \mathrm{~g}$ ), a FRF se caracteriza como um produto com alto teor de fibras. 
O teor de carboidratos totais da FRF foi 81,40 g/100g, valor próximo ao reportado por Sganzerla et al. (2020), que encontraram 79,82 g/100g para a farinha da casca, ao realizarem a caracterização de resíduos de feijoa para sua incorporação em embalagem biodegradável. De acordo com Almeida et al. (2020), os principais monossacarídeos da casca da feijoa são a glicose e a xilose, seguidos do ácido urônico, o qual estaria relacionado à presença de pectina.

Tabela 2. Caracterização físico-química e composição de minerais da farinha de resíduos do despolpamento de feijoa.

\begin{tabular}{ll}
\hline \multicolumn{1}{c}{ Parâmetros* } & \multicolumn{1}{c}{ Resultados } \\
\hline Umidade $(\mathrm{g} / 100 \mathrm{~g})$ & $11,33 \pm 0,35$ \\
Cinzas $(\mathrm{g} / 100 \mathrm{~g})$ & $2,31 \pm 0,06$ \\
Lipídeos totais $(\mathrm{g} / 100 \mathrm{~g})$ & $1,27 \pm 0,10$ \\
Proteína bruta $(\mathrm{g} / 100 \mathrm{~g})$ & $3,70 \pm 0,11$ \\
Fibra alimentar total $(\mathrm{g} / 100 \mathrm{~g})$ & $40,50 \pm 0,14$ \\
Carboidratos totais $(\mathrm{g} / 100 \mathrm{~g})$ & $81,40 \pm 0,33$ \\
Carotenoides totais $(\mu \mathrm{g} / 100 \mathrm{~g})$ & $3353,09 \pm 19,87$ \\
Ácido ascórbico $(\mathrm{mg} / 100 \mathrm{~g})$ & $265,63 \pm 18,40$ \\
$\mathrm{pH}$ & $2,85 \pm 0,02$ \\
Acidez titulável $(\mathrm{g} \mathrm{de} \mathrm{ácido} \mathrm{cítrico/100g)}$ & $15,12 \pm 0,07$ \\
$\mathrm{~N}(\mathrm{mg} / \mathrm{kg})$ & $8000,00 \pm 565,69$ \\
$\mathrm{P}(\mathrm{mg} / \mathrm{kg})$ & $633,33 \pm 57,74$ \\
$\mathrm{~K}(\mathrm{mg} / \mathrm{kg})$ & $4550,00 \pm 353,55$ \\
$\mathrm{Ca}(\mathrm{mg} / \mathrm{kg})$ & $650,00 \pm 70,71$ \\
$\mathrm{Mg}(\mathrm{mg} / \mathrm{kg})$ & $300,00 \pm 0,00$ \\
$\mathrm{~S}(\mathrm{mg} / \mathrm{kg})$ & $1266,67 \pm 57,74$ \\
$\mathrm{Fe}(\mathrm{mg} / \mathrm{kg})$ & $15,10 \pm 0,28$ \\
$\mathrm{Mn}(\mathrm{mg} / \mathrm{kg})$ & $6,65 \pm 0,35$ \\
$\mathrm{Cu}(\mathrm{mg} / \mathrm{kg})$ & $3,15 \pm 0,21$ \\
$\mathrm{Zn}(\mathrm{mg} / \mathrm{kg})$ & $1,05 \pm 0,07$ \\
\hline
\end{tabular}

*Valor médio \pm desvio padrão $(\mathrm{n}=3)$, em base seca. Fonte: Autores (2021).

Quanto aos carotenoides totais, a FRF apresentou um teor de 3353,09 $\mu \mathrm{g} / 100 \mathrm{~g}$, valor esse superior ao da farinha apenas do mesocarpo da feijoa, 2170,00 $\mu \mathrm{g} / 100 \mathrm{~g}$, encontrado no estudo de Almeida et al. (2020). Os carotenoides desempenham um papel potencialmente importante na saúde humana, atuando como antioxidantes biológicos, protegendo células e tecidos dos efeitos nocivos dos radicais livres e do oxigênio singlete e são usados como corantes naturais na indústria de alimentos (Oreopoulou \& Tzia, 2007).

A feijoa é considerada uma boa fonte de vitamina C, com valores médios de $32,9 \mathrm{mg} / 100 \mathrm{~g}$ na fruta in natura (Weston, 2010; Zhu, 2018). Amarante et al. (2017a), analisando a casca e a polpa de frutos in natura de feijoa, verificaram teores de ácido ascórbico variando de 63,5 a 101,0 mg/100g para casca e de 38,7 a 92,5 mg/100g para polpa. A FRF apresentou um teor de 265,63 mg/100g de ácido ascórbico, valor esse superior ao das farinhas de resíduos de laranja (145,83 mg/100g) (Clemente et al., 2012) e de goiaba (45,06 mg/100g) (Soares et al, 2017).

Os frutos de feijoa são caracterizados pela sua acidez, com pH variando de 2,0 a 3,5 (Amarante et al, 2013). Parra e Fischer (2013) afirmam que o pH pós-colheita da feijoa varia entre 3,1 e 3,5, o qual depende da cultivar, de fatores 
agroecológicos e de condições de armazenamento. A partir da remoção da água, em decorrência do processo de secagem, ocorre a concentração dos constituintes, incluindo os ácidos orgânicos, o que contribui para a redução do valor do $\mathrm{pH}$ da farinha de resíduos de feijoa $(2,85)$ e o aumento da acidez titulável $(15,12 \mathrm{~g}$ de ácido cítrico/100g).

Com relação aos macronutrientes (mg/kg), observa-se que a FRF apresentou maiores valores de N (8000), K (4550) e S (1266,67), seguidos de Ca (650), P (633,33) e Mg (300). Já os micronutrientes em maiores concentrações foram Fe $(15,10$ $\mathrm{mg} / \mathrm{kg}$ ) e Mn (6,65 mg/kg). Esses resultados estão de acordo com os apresentados por Amarante et al. (2019) para a casca in natura de diferentes genótipos de feijoa. Diante da sua composição mineral, a FRF se mostra como uma importante e potencial fonte de elementos essenciais.

\subsection{Características tecnológicas e cor instrumental dos cookies}

A Tabela 3 apresenta os resultados das características tecnológicas dos biscoitos tipo cookies. De acordo com Assis et al. (2009), um alto percentual de perda de peso com o assamento é decorrente da menor retenção de água e afeta o rendimento dos biscoitos. Ferreira et al. (2020) avaliando cookies sem glúten contendo farinha de linhaça e enriquecido com fibras obtiveram valores entre 10,45 e 11,51\%, valores inferiores aos observados para as amostras de cookies com FRF (13,08 $15,79 \%$ ), o que pode ser atribuído ao conteúdo de proteínas e fibras presentes na farinha de linhaça, contribuindo ao aumento da capacidade de retenção de água dos biscoitos (Ganorkar \& Jain, 2014).

Não foram verificadas diferenças significativas $(\mathrm{p} \geq 0,05)$ no fator de expansão das amostras analisadas. Biscoitos com fator de expansão muito alto ou muito baixo causam problemas na indústria, resultando em produtos com tamanho pequeno ou peso muito elevado (Rasper, 1991). A farinha ou qualquer outro ingrediente, os quais absorvem água durante a mistura da massa, poderão reduzir o fator de expansão (Odorica-Falomir \& Paredes-Lópes, 1991).

Tabela 3. Características tecnológicas dos biscoitos tipo cookies elaborados com diferentes concentrações de farinha de resíduos do despolpamento de feijoa.

\begin{tabular}{lcc}
\hline Amostras de cookies & Perda de peso (\%) & Fator de expansão \\
\hline Padrão & $13,08 \pm 0,16^{\mathrm{b}}$ & $5,78 \pm 0,07^{\mathrm{a}}$ \\
F1 & $15,79 \pm 0,36^{\mathrm{a}}$ & $5,89 \pm 0,11^{\mathrm{a}}$ \\
F2 & $15,20 \pm 0,21^{\mathrm{a}}$ & $5,92 \pm 0,20^{\mathrm{a}}$ \\
\hline
\end{tabular}

Valores expressos como média \pm desvio padrão seguidos por letras diferentes, nas colunas, que indicam diferença estatística significativa entre os tratamentos, em nível de 5\% pelo teste de Tukey. Legenda: Padrão (0\% de FRF), F1 (10\% de FRF) e F2 (20\% de FRF). Fonte: Autores (2021).

Considerando os resultados obtidos para os parâmetros de cor (Tabela 4), é possível observar que os valores de L* (luminosidade) dos cookies com adição de FRF foram menores $(\mathrm{p}<0,05)$ em relação à amostra padrão, com redução deste parâmetro à medida que se aumentou a \% de FRF na formulação. De acordo com De Toledo et al. (2017), a cor dos cookies é formada no cozimento, devido à reação de Maillard, sendo que nos cookies produzidos com adição de farinha de resíduos de frutas a cor é influenciada pelo tipo de farinha adicionada à massa. Isso pode ser observado pela diferença significativa $(\mathrm{p}<0,05)$ entre os valores de $\mathrm{a}^{*}$ (intensidade de vermelho) e $\mathrm{b} *$ (intensidade de amarelo) encontrados para a amostra padrão e as amostras F1 e F2.

Em relação aos valores de $\Delta \mathrm{E}$, as amostras $\mathrm{F} 1(6,00)$ e F2 $(12,85)$ apresentam valores de $\Delta \mathrm{E}$ superiores a 5 , logo o observador consegue visualizá-las como cores totalmente distintas quando comparadas a amostra padrão (Mokrzycki \& Tatol, 2011), sendo o $\Delta \mathrm{E}$ de maior valor verificado para a $\mathrm{F} 2$, decorrente da adição superior de FRF (20\%) na formulação deste cookie e consequente incremento na sua cor. 
Tabela 4. Parâmetros de cor dos biscoitos tipo cookies elaborados com diferentes concentrações de farinha de resíduos do despolpamento de feijoa.

\begin{tabular}{lcccc}
\hline Amostras de cookies & $\mathbf{L}^{*}$ & $\mathbf{a}^{*}$ & $\mathbf{b}^{*}$ & $\Delta \mathbf{E}$ \\
\hline Padrão & $50,96 \pm 0,60^{\mathrm{a}}$ & $0,80 \pm 0,08^{\mathrm{c}}$ & $19,81 \pm 0,47^{\mathrm{b}}$ & 0,00 \\
F1 & $45,40 \pm 0,26^{\mathrm{b}}$ & $2,33 \pm 0,05^{\mathrm{b}}$ & $21,46 \pm 0,14^{\mathrm{a}}$ & 6,00 \\
F2 & $38,90 \pm 0,08^{\mathrm{c}}$ & $4,58 \pm 0,04^{\mathrm{a}}$ & $22,12 \pm 0,36^{\mathrm{a}}$ & 12,85 \\
\hline
\end{tabular}

Valores expressos como média \pm desvio padrão seguidos por letras diferentes, nas colunas, que indicam diferença estatística significativa entre os tratamentos, em nível de 5\% pelo teste de Tukey. Legenda: Padrão (0\% de FRF), F1 (10\% de FRF) e F2 (20\% de FRF). Fonte: Autores (2021).

\subsection{Análise sensorial}

A Tabela 5 apresenta os resultados da análise sensorial dos cookies, e é possível observar que em todos os parâmetros as amostras com FRF diferiram estatisticamente da amostra padrão ( $\mathrm{p}<0,05)$, exceto para a aparência. Quanto ao aroma, as formulações com a incorporação da FRF foram mais aceitas (7,65 para F1 e 7,71 para F2) em comparação com a amostra padrão $(7,16)$, visto que a feijoa se caracteriza pelo aroma agradável e marcante, relacionado a presença de compostos voláteis, como terpenos, taninos, quinonas, flavonoides e outros (Sun-Waterhouse et al., 2013). Em relação à textura, observa-se que as fibras presentes na FRF podem ter contribuído positivamente nesse parâmetro, sendo que a amostra F2, com 20\% de FRF, teve a maior média $(8,07)$ atribuída pelos provadores.

Tabela 5. Análise sensorial de aceitação e intenção de compra dos biscoitos tipo cookies com diferentes concentrações de farinha de resíduos do despolpamento de feijoa.

\begin{tabular}{lccc}
\hline Teste de aceitabilidade & Padrão & F1 & F2 \\
\hline Aparência & $7,64 \pm 1,02^{\mathrm{a}}$ & $7,45 \pm 1,07^{\mathrm{a}}$ & $7,78 \pm 0,90^{\mathrm{a}}$ \\
Aroma & $7,16 \pm 1,12^{\mathrm{b}}$ & $7,65 \pm 0,97^{\mathrm{a}}$ & $7,71 \pm 0,96^{\mathrm{a}}$ \\
Sabor & $7,47 \pm 1,07^{\mathrm{b}}$ & $8,11 \pm 0,81^{\mathrm{a}}$ & $8,18 \pm 0,84^{\mathrm{a}}$ \\
Textura & $7,38 \pm 1,47^{\mathrm{b}}$ & $7,85 \pm 0,89^{\mathrm{ab}}$ & $8,07 \pm 1,00^{\mathrm{a}}$ \\
Aceitação global & $7,31 \pm 1,18^{\mathrm{b}}$ & $8,04 \pm 0,84^{\mathrm{a}}$ & $7,95 \pm 0,93^{\mathrm{a}}$ \\
Índice de aceitabilidade $(\%)$ & 82,36 & 86,28 & 88,17 \\
\hline Teste de intenção de compra & $2,29 \pm 0,85^{\mathrm{a}}$ & $1,76 \pm 0,79^{\mathrm{b}}$ & $1,65 \pm 0,89^{\mathrm{b}}$ \\
\hline
\end{tabular}

Valores expressos como média \pm desvio padrão seguidos por letras diferentes, nas linhas, que indicam diferença estatística significativa entre os tratamentos, em nível de 5\% pelo teste de Tukey. Legenda: Padrão (0\% de FRF), F1 (10\% de FRF) e F2 (20\% de FRF). Fonte: Autores (2021).

Observa-se que os cookies com FRF tiveram maiores médias de aceitação global (p<0,05), sendo que a amostra F2, com 20\%, apresentou o maior índice de aceitabilidade (88,17\%). De acordo com Dutkosky (2007), para um produto ser considerado "aceitável” deve apresentar, no mínimo, 70\% de aceitabilidade.

Todas as médias das notas atribuídas pelos provadores no teste de aceitabilidade, para as amostras F1 e F2, foram próximas de 8 na escala hedônica, demonstrando que os provadores "gostaram muito" dos cookies com FRF.

O teste de intenção de compra reproduziu a aceitabilidade dos provadores, sendo que as médias das notas para as amostras corresponderam a "certamente compraria" e "provavelmente compraria" na escala hedônica de intenção de compra. Além disso, os cookies com FRF diferiram estatisticamente $(\mathrm{p}<0,05)$ da amostra padrão. 
Comparativamente com outros estudos de incorporação de farinha de resíduos de frutas em cookies, observa-se que a FRF contribuiu positivamente para a aceitação das amostras nas concentrações de 10 e $20 \%$, o que pode estar relacionado ao fato desta farinha apresentar sabor e aroma diferenciados, além da característica ácida marcante.

Aquino et al. (2010) obtiveram escores médios menores, entre 4 e 5, numa escala hedônica de 9 pontos, para cookies elaborados com 10 e $20 \%$ de farinha de resíduos de acerola. Ramos et al. (2020) desenvolveram cookies com substituição parcial de 15\% de farinha de trigo por diferentes farinhas de resíduos de frutas (casca de abacaxi, casca de manga, casca de banana, casca de laranja, entrecasca de melancia e amêndoa da manga) e para os parâmetros sensoriais de aparência e sabor foram encontrados valores médios entre 6 e 7. Silva et al. (2019) observaram menor aceitação, principalmente da textura e do sabor, dos cookies elaborados com farinha de caroço de abacate a partir da concentração de $20 \%$.

Oliveira, Rosa e Aquino (2021), realizando a aceitação sensorial de muffins com substituição parcial da farinha de trigo pela FRF, observaram que a farinha em estudo contribuiu positivamente na aceitabilidade e na intenção de compra de muffins, sendo que todas as médias das notas atribuídas pelos provadores no teste de aceitabilidade para as amostras com $10 \mathrm{e}$ $20 \%$ de FRF foram superiores a 7 na escala hedônica, e obtiveram índices de aceitabilidade maiores que $85 \%$.

Esses resultados reforçam a promissora aplicação de farinha de resíduos de feijoa, substituindo parcialmente a farinha de trigo em produtos de panificação, com o aproveitamento de partes da fruta geralmente descartadas, diversificando a oferta e contribuindo ainda para incrementar o valor nutricional desses produtos.

\section{Conclusão}

Este estudo demonstrou a potencialidade do aproveitamento dos resíduos do despolpamento da feijoa como farinha, considerando sua relevante composição nutricional e utilização na substituição parcial da farinha de trigo para elaboração de biscoitos tipo cookies.

A FRF contribuiu positivamente na aceitabilidade e na intenção de compra dos cookies, os quais tiveram maiores médias de aceitação global em relação à amostra padrão, sendo que a amostra F2, com 20\%, apresentou o maior índice de aceitabilidade. Os resultados obtidos neste estudo evidenciam a potencialidade da utilização da FRF, podendo contribuir para a diversificação da oferta de produtos, além de agregar valor ao resíduo do processamento de uma fruta nativa com potenciais características nutricionais.

No entanto, verifica-se a necessidade de estudos futuros, considerando maiores \% de substituição da FRF pela farinha de trigo, visando melhorar a qualidade nutricional dos produtos, além da realização da caracterização físico-química dos produtos finais.

\section{Agradecimentos}

Os autores agradecem a Empresa de Pesquisa Agropecuária e Extensão Rural de Santa Catarina (EPAGRI) de São Joaquim-SC pelo fornecimento das amostras de feijoa e ao IFSC pelo fomento e incentivo à pesquisa (Edital $\left.\mathrm{n}^{\circ} 10 / 2019 / \mathrm{PROPPI}\right)$.

\section{Referências}

Almeida, J. dos S. O. de, Dias, C. O., Arriola, N. D. A., Freitas, B. S. M. de, Francisco, A. de., Petkowicz, C. L. O., Araujo, L., Guerra, M. P., Nodari, R. O., \& Amboni, R. D. C. (2020). Feijoa (Acca sellowiana) peel flours: A source of dietary fibers and bioactive compounds. Food Bioscience, 38 , 100789. https://doi.org/10.1016/j.fbio.2020.100789

Amarante, C. V. T. do, Souza, A. G. de, Beninca, T. D. T., \& Steffens, C. A. (2017a). Fruit quality of Brazilian genotypes of feijoa at harvest and after storage. Pesquisa Agropecuária Brasileira, 52(9), 734-742. https://doi.org/10.1590/s0100-204x2017000900005 
Amarante, C. V. T. do, Souza, A. G. de, Beninca, T. D. T., \& Steffens, C. A. (2017b). Phenolic content and antioxidant activity of the fruit in Brazilian genotypes of feijoa. Pesquisa Agropecuária Brasileira, 52(12), 1223-1230. https://doi.org/10.1590/s0100-204x2017001200011

Amarante, C. V. T. do, Souza, A. G. de, Steffens, C. A., \& Beninca, T. D. T. (2019) Centesimal and mineral composition of the fruit in Brazilian genotypes of feijoa (Acca sellowiana). Revista Brasileira de Fruticultura, 41(6), 1-7. https://doi.org/10.1590/0100-29452019487

American Association of Cereal Chemists - AACC (2000). Approved methods. 10 ed. Saint Paul, 2 v.

Aquino, A. C. M. de S., Móes, R. S., Leão, K. M. M., Figueiredo, A. V. D., \& Castro, A. A. (2010). Avaliação físico-química e aceitação sensorial de biscoitos tipo cookies elaborados com farinha de resíduos de acerola. Revista do Instituto Adolfo Lutz, 69(3), 379-386. http://www.ial.sp.gov.br/resources/insitutoadolfo-lutz/publicacoes/rial/10/rial69_3_completa/1303.pdf

Arioli, C. J., Pinto, F. A. M. F., Araujo, L., Santos, K. dos, Ciotta, M. N., \& Pasa, M. da S. (org.). (2018). A cultura da goiabeira-serrana. Florianópolis: Epagri, 215p.

Assis, L. M., Zavareze, E. R., Radunz, A. L., Dias, A. R. G., Gutkoski, L. C., \& Elias, M. C. (2009). Propriedades nutricionais, tecnológicas e sensoriais de biscoitos com substituição de farinha de trigo por farinha de aveia ou farinha de arroz parboilizado. Alimentos e Nutrição, 20(1), 15-24. http://servbib.fcfar.unesp.br/seer/index.php/alimentos/article/view/944/771

Association of Official Analytical Chemists - AOAC. (1984). Official methods of analysis of the association of analytical chemists. Arlington: Sidney Williams.

Association of Official Analytical Chemists - AOAC. (2005). Official methods of analysis of the association of analytical chemists. USA: AOAC International, 18 ed.

Ayala-Zavala J.F., Vega-Veja, V., Rosas-Domínguez, C., Palafox-Carlos, H., Villa-Rodriguez, J. A., Siddiqui, M. W., Dávila-Aviña, J. E., \& GonzálezAguilar, G. A. (2011). Agro-industrial potential of exotic fruit byproducts as a source of food additives. Food Research International, 44, 1866-1874. http://dx.doi.org/10.1016/j.foodres.2011.02.021

Benassi, M. T., \& Antunes, A. J. (1988). A comparison of metaphosphoric and oxalic acids as extractants solutions for the determination of vitamin C in selected vegetables. Arquivos de Biologia e Tecnologia, 31(4), 507-513.

Brasil. (2003). Resolução n ${ }^{\circ}$ 359, de 23 de dezembro de 2003. Aprova o Regulamento Técnico de Porções de Alimentos Embalados para Fins de Rotulagem Nutricional. Agência Nacional de Sanitária ANVilância http://antigo.anvisa.gov.br/documents/10181/2718376/RDC_359_2003_COMP.pdf/1e860ef6-10e6-404b-81e2-87aae8cfd53a

Brasil. (2005). Resolução $\mathrm{n}^{\circ}$ 263, de 22 de setembro de 2005. Regulamento técnico para produtos de cereais, amidos, farinhas e farelos. Agência Nacional de Vigilância Sanitária (ANVISA).

Brasil. (2012). Resolução $n^{\circ}$ 54, de 12 de novembro de 2012. Dispõe sobre o Regulamento Técnico sobre Informação Nutricional Complementar. Agência Nacional de Vigilância Sanitária (ANVISA).: http://antigo.anvisa.gov.br/documents/10181/4825974/\%281\%29RDC_54_2012_.pdf/921d3c25-cef9-40d89b3f-7861eb7b8235

Clemente, E., Flores, A. C., Rosa, C. I. L. F., \& Oliveira, D. M. (2012). Características da Farinha de Resíduos do Processamento de Laranja. Revista Ciências Exatas e Naturais, 14 (2), 257-269. https://doi.org/10.5935/RECEN.2012.02.06

De Toledo, N. M. V., Nunes, L. P., da Silva, P. P. M., Spoto, M. H. F., \& Canniatti-Brazaca, S. G. (2017). Influence of pineapple, apple and melon byproducts on cookies: physicochemical and sensory aspects. International Journal of Food Science and Technology, 52(5), 1185-1192. https://doi.org/10.1111/ijfs.13383

Diettrich, L., Bauer, V. F., \& De Oliveira, E. G. (2016). Elaboração de biscoito integral tipo cookie a partir de farinha de bagaço de uva. XXV Congresso Brasileiro de Ciência e Tecnologia de Alimentos: Gramado. http://www.ufrgs.br/sbctars-eventos/xxvcbcta/anais/files/874.pdf

Dutkosky, S. D. (2007). Análise sensorial de alimentos. Curitiba: Champagnat, 2 ed.

Ferreira, F.J.N, Alves, R.A., Sousa, A.M.B, Abreu, V.K.G, Firmino, F., Lemos, T.O., \& Pereira, A.L.F. (2020). Physico-chemical and sensory characteristics of gluten free cookies containing flaxseed flour and enriched with fiber. Research, Society and Development, 9(7): 1-17, e565974474. http://dx.doi.org/10.33448/rsd-v9i7.4474

Ganorkar, P., \& Jain, R. (2014). Effect of flaxseed incorporation on physical, sensorial, textural and chemical attributes of cookies. International of Food Research Journal, 21(4), 1515-1521. http://www.ifrj.upm.edu.my/21\%20(04)\%202014/35\%20IFRJ\%2021\%20(04)\%202014\%20Ganorkar\%20110.pdf

González-García, K. E., Guerra-Ramírez, Diana, Ángel-Coronel, O. A. del, \& Cruz-Castillo, J. G. (2017). Physical and chemical attributes of feijoa fruit in Veracruz, Mexico. Revista Chapingo Serie Horticultura, 24(1), 5-12. http://dx.doi.org/10.5154/r.rchsh.2017.01.006

Instituto Adolfo Lutz - IAL. (2008). Métodos físico-químicos para análise de Alimentos. São Paulo: Instituto Adolfo Lutz, 4 ed.

Lima, J. P., Portela, J. V. F., Marques, L. R., Alcântara, M. A., \& El-Aouar, Â. A. (2015). Farinha de entrecasca de melancia em biscoitos sem glúten. Ciência Rural, 45(9), 1688-1694. https://doi.org/10.1590/0103-8478cr20130209

Monforte, M. T., Lanuzza, F., Mondello, F., Naccari, C., Pergolizzi, S., \& Galati, E. M. (2014). Phytochemical compositon and gastroprotective effect of Feijoa sellowiana Berg. fruit from Sicily. Journal of Coastal Life Medicine, 2(1), 14-21. http://doi.org/10.12980/JCLM.2.2014J12

Mokrzycki, W., \& Tatol, M. (2011). Color difference Delta E - A survey. Machine Graphics and Vision, 20(4), 383-411. https://www.researchgate.net/publication/236023905_Color_difference_Delta_E_-_A_survey 
Odorica-Falomir, C., \& Paredes-López, O. (1991). Effect of safflower protein isolates on cookie characteristics. International Journal Food Science and Technology, 26(1), 39-43. https://ifst.onlinelibrary.wiley.com/doi/epdf/10.1111/j.1365-2621.1991.tb01139.x

Oliveira, P. M. de, Rosa, B. R. S., \& Aquino, A. C. M. de S. (2021). Farinha de resíduos de feijoa (Acca sellowiana): propriedades tecnológicas e aceitação sensorial de muffins. Brazilian Journal of Development, 7(3), 26577-26586. https://doi.org/10.34117/bjdv7n3-383

Oreopoulou, V., \& Tzia, C. (2007). Utilization of plant by-products for the recovery of proteins, dietary fibers, antioxidants, and colorants. Utilization of ByProducts and Treatment of Waste in the Food Industry, 209-232. https://doi.org/10.1007/978-0-387-35766-9_11

Parra, C. A., \& Fischer, G. (2013). Maduración y comportamiento poscosecha de la feijoa (Acca sellowiana (O. Berg) Burret). Una revisión. Revista Colombiana de Ciencias Hortícolas, 7(1), 98-110. https://doi.org/10.17584/rcch.2013v7i1.2039

Ramos, A. A., Pereira, R.das D., Andressa, I., Achmiele, M., \& Amaral, T. N. (2020). Desenvolvimento de cookies com coprodutos de frutas. Research, Society and Development, 9(10), 1-16. http://dx.doi.org/10.33448/rsd-v9i10.8918

Rasper, V. F. (1991). Quality evaluation of cereal and cereal products. In: Lorenz, K. J., \& Kulp, K. (Ed.). Handbook of cereal science and technology. New York: Marcel Dekker, 595-638.

Rodriguez-Amaya, D. B. (1999). A guide to carotenoid analysis in foods. USA: International Life Sciences Institute (ILSI) Press. 71 p.

Santos, P. H., Ribeiro, D. H. B., Micke, G. A., Vitali, L., \& Hense, H. (2019). Extraction of bioactive compounds from feijoa (Acca sellowiana (O. Berg) Burret) peel by low and high-pressure techniques. The Journal of Supercritical Fluids, 145, 219-227. https://doi.org/10.1016/j.supflu.2018.12.016

Sganzerla, W. G., Rosa, G. B., Ferreira, A. L., Rosa, C. G. da, Beling, P. C., Xavier, L. O., Hansen, C. M., Ferrareze, J. P., Nunes, M. R., Barreto, P. L. M., \& Veeck, A. P. de. (2020). Bioactive food packaging based on starch, citric pectin and functionalized with Acca sellowiana waste by-product: Characterization and application in the postharvest conservation of apple. International Journal of Biological Macromolecules, 147, 295-303. https://doi.org/10.1016/j.ijbiomac.2020.01.074

Silva, F. C. da. (2009). Manual de análises químicas de solos, plantas e fertilizantes. Brasília: Embrapa. 2 ed.

Silva, I. G. da, Andrade, A. P. C. de, Silva, L. M. R. da, \& Gomes, D. S. (2019). Elaboração e análise sensorial de biscoito tipo cookie feito a partir da farinha do caroço de abacate. Brazilian Journal of Food Technology, 22, 1-10. https://doi.org/10.1590/1981-6723.20918

Soares, D. J., Diogenes, A. de M. G., Neto, L. G. de M., Costa, Z. R. T., Alves, V. R., \& Santos, M. G. (2017). Utilização de farinha de resíduos de goiaba na elaboração de pães. Revista CIENTEC, 9(1), 97-103. http://revistas.ifpe.edu.br/index.php/cientec/article/view/39/32

Soquetta, M. B., Stefanello, F. S., Huerta, K. D. M., Monteiro, S. S., Da Rosa, C. S., \& Terra, N. N. (2016). Characterization of physiochemical and microbiological properties, and bioactive compounds, of flour made from the skin and bagasse of kiwi fruit (Actinidia deliciosa). Food Chemistry, 199, 471478. https://doi.org/10.1016/j.foodchem.2015.12.022

Sun-Waterhouse, D., Wang, W., Waterhouse, G. I. N., \& Wadhwa, S. S. (2013). Utilisation Potential of Feijoa Fruit Wastes as Ingredients for Functional Foods. Food Bioprocess Technology, 6, 3441-3455. https://doi.org/10.1007/s11947-012-0978-3

Weston, R. J. Bioactive products from fruit of the feijoa (Feijoa sellowiana, Myrtaceae): A review. (2010). Food Chemistry, 121(1), 923-926. https://doi.org/10.1016/j.foodchem.2010.01.047

Zhu, F. (2018) Chemical and biological properties of feijoa (Acca sellowiana). Trends in Food Science \& Technology, 81, 121-131. https://doi.org/10.1016/j.tifs.2018.09.008 